\title{
Comment, toi aussi...
}

\author{
Lise Gauvin
}

\begin{abstract}
What? You too?
(This in the form of a conversation between two women.) I confess, I' $m$ capitulating. You can't be a writer any longer if you don't writenovels. Other perfectly respectable forms are disguising themselves as fiction these days. Although, contrary to appearances, I wonder if one doesn't hide oneself more in so-called personal forms. Maybe. As soon as you write 'he' followed by the imperfect tense, people are willing to believe anything. It's clear that the novel represents power. I've always thought it suited males, because you have to take yourself for God. But who said God was a man? Hang on, I'm not saying women can't do it. But first they have to constitute themselves as subject. What I would like is just to take everything in my everday life and call that 'novel.' You just have to take what you see and hear. Okay, okay, I'm leaving before I end up between two covers.
\end{abstract}

-Comment, toi aussi?

-Jecapitule, jel'avoue.

-C'est vrai, $c^{\prime}$ est vraiment vrai?

- Rien de plus sérieux. Je commence déjà mon deuxième chapitre. Tu n'en sauras pas plus pour l'instant. En tout cas, ma décision est prise. C'est déjà quelque chose.

- Ça alors! Après avoir tant déblatéré contre l'impérialisme du roman.

- Exactement. Et je n'ai pas fini de me révolter. On n'entend parler que de cela aujourd'hui. N'est écrivain que celui qui a osé qualifier sa prose de 'roman', étiquette magique et 'Sésame, ouvre-toi' de toutes les tribunes et de tous les cabinets de lecture contemporains. A tel point que biographies, mémoires et autres textes réputés sérieux- 
même l'essai - se travestissent en cette forme garante de toutes les séductions. Un écrivain connu m'expliquait récemment qu'il fallait gauchir le genre, ruser avec lui, installer l'ambiguïté au coeur même de l'événement historique et daté. Le terme de 'fiction', loin de disqualifier les textes, lui ajoute une sorte de plus-value. C'est un passeport garanti, un voyage organisé. La moitié des gens que je connais écrit des romans, l'autre moitié ne lit que cela. Alors j'ai fini par comprendre ...

- C'est bien ce que je disais. Tu capitules. En tout cas, moi, je résiste. Je préfère des formes plus franches, comme le journal ou la lettre. On sait qui parle au moins. C'est du direct. Pas de faux-fuyant.

- Je me demande si on ne se cache pas encore plus dans des formes dites personnelles. Parce que le jeétant mis à nu, on ne peut faire autrement que de le modifier un tant soit peu, delui faire une toilette pourla visite. Un petit lifting inconscient, quoi!

- Peut-être, mais l'opération se fait en toute liberté. La lettre est un genre caméléon alors que la fiction est une terrible contrainte, quoi qu' on dise. Dès qu' on a le malheur d'écrire 'il' suivi d'un imparfait, le lecteur est prêt à croire tout ce qu'on dit, à prendre nos phrases pour del' argent comptant, à nous en vouloirsi on fait un faux pas, si on oublie un personnage dans un coin, si on ne donne pas toute l'ampleur qu'il faut aux événements. Selon une logique implacable, à peine les premiers mots sont-ils posés, qu'ils doivent correspondre à l'image que le lecteur se fait de la vraie vie. Il faut à la fois le satisfaire et l'étonner. Ça nous fait une belle jambe! N'est-ce pas toi-même d'ailleurs qui criait très fort que le roman est une entreprise globalisante, voire totalisante?

- Ce qui est sûr, $c^{\prime}$ est que le roman est pouvoir. Ça, $c^{\prime}$ est pas moi qui l'ai déclaré. Je l'ai entendu dire l'autre jour à la télé, par un poète qui s'était mis à la tâche lui aussi. Comme tout le monde. 'Que voulezvous dire', lui avait demandél'interviewer étonné? 'Que je peux, à ma guise, donner toutes les qualités et tous les défauts que je veuxà tel ou tel personnage, dire de vous - ou de votre double - que vous êtes laid et bête, si cela me plaît. Cela ne dépend que de moi' , avait-il précisé. Il avait même ajouté qu'il souhaitait à chacun d'écrire son roman. Que cela était un excellent exercice de santé. Sûrement aussi bon que l'aérobique. La réponse ne m'avait pas tout à fait satisfaite. Je suis allée le rejoindre un samedi après-midi, lors d'une rencontre à une librairie. J'ai dit: 'Le roman est pouvoir parce qu'il est totalité, système 
d'explication du monde?' Il a éludé la question. Il n'aime pas les phrases abstraites. 'Le roman est le seul pouvoir', a-t-il répliqué. 'Cela dépend des lecteurs', ai-je dit. 'En un ou deux mots', m'a-t-il ciemandé en riant. Cette fois, c'était l'ancien ministre qui parlait. Je suis revenue à la charge. 'Le roman est pouvoir seulement s'il est fermé, s'il est une histoire avec un début, un milieu, et une fin?' Alors il m'a fait cette confidence. 'Le mien est justement pas cela et c'est la raison pour laquelle l'éditeur le refuse. Il veut que je le refasse, que je change beaucoup de choses.' Tu vois comme c'est compliqué.

- Moi, j'ai toujours pensé que le roman convenait mieux aux hommes, malgré tout. Parce que, quelque part, il faut se prendre pour Dieu.

- Depuis quand Dieu est-il mâle? Et Madame de la Fayette, et Madame Woolf, et Madame Duras, et Madame Roy, qu'est-ce quet'en fais?

- Doucement, là, c'est pas nécessaire de monter sur tes grands chevaux. J'ai pas dit que c'était une forme mâle. Je m'en garde bien. Je ne tombe pas dans l'erreur naïve de croire aux formes sexuées. Ça se passe davantage au niveau de la pré-écriture. Au niveau dela disposition mentale préalable à toute écriture. Quand une femme s'y met, il arrive qu'elle s'en tire assez bien, parce qu'il y a chez elle une grande capacité d'attention aux mille petites choses qui font la texture même du roman. Mais ce que je veux dire, $c^{\prime}$ est qu'elle doit d'abord accomplir un long travail sur elle-même. Ecrire un roman, $c^{\prime}$ est se constituer comme sujet - ce qui est le degré zéro du personnage-, se hausser d'un cran ou deux au-dessus de la mêlée, et ensuite jouer avec son je comme avec un dé.

-Moi,j'ai toujours cru quec'était avant tout une recherche.

-Oui, pendant, mais avant de commencer, il faut se convaincre que le regard que l'on porte sur le monde, le temps qu'on le porte et selon un jugement subjectif absolu, est la chose la plus importante au monde.

-Maisily a roman et roman...

- Ecrire une histoire, c'est toujours jusqu'à un certain point choisir de faire l'Histoire. Ça suppose une bonne dose de confiance, de prétention et de témérarité. C'est pas évident au départ. Heureusement pour moi, je n'éprouve pas ce besoin-là. Et puisque tu veux tout savoir, le roman a un petit côté documentaire qui m'agace. Personnellement, j'aime mieux lire Historia ou le Geographic magazine. Ça 
me suffit pour le rêve réalisable. Ou la fiction existe, ou elle n'existe pas, non? J'en connais qui ont ratissé des bibliothèques pendant des années avant d'écrire leur roman. Que de temps perdu.

- Les romans historiques, moi aussi ça m' ennuie. Je me demande si $c^{\prime}$ est pas le folklore des temps modernes.

- Ce que j'aimerais, si jamais je m'y mettais - et je t'assure que c'est pas encore fait $-c^{\prime}$ est ramasser tout ce qui me tombe sous la main, coller des billets de théâtre à côté de réflexions sur le sens de la vie, reproduire en vrac faits divers, listes d'épicerie, projets de vacances, réunions de comités et intituler cela ensuite: roman. Ce serait un vrai fourre-tout.

- Dommage, $c^{\prime}$ est déjà fait. $C^{\prime}$ est vexant. Dès que tu penses à une forme, si tu as lu le moindrement, tu t'aperçois qu'elle existe déjà. On dit d'ailleurs que la littérature est un vaste plagiat, sauf la première, qui a jamais été trouvée.

- La plupart des gens qui écrivent nes'embarrassent pas de tousces scrupules.

-Ou ils acceptent modestement de refaire, de reprendre inlassablement les mêmes motifs, de regarder indéfiniment la même chose, mais sous tous ses angles, par devant, par derrière ou de côté, commel'oeil de la mouche. Le romancier est un pervers polymorphe. J'ai oublié qui a dit ça.

- Capable de tout voir, donc voyeur, donc omniscient.

- Voyeur, oui, mais en même temps myope. L'oeil de la mouche ne perçoit qu'un petit morceau de réel à la fois, même s'il le voit de tous les côtés.

-Etalors?

- Alors c'est simple. Tu n'as qu'à décrire ce qui arrive dans ton champ de vision, devant, derrière et à côté de toi. Ou dans ton champ d'audition.

- Bon, bon. J'ai compris. Je te laisse. Avant de me retrouver entre deux couvertures cartonnées. 